\title{
A intervenção fisioterapêutica na reabilitação pós cirurgia de redesignação de sexo masculino para feminino: relato de caso
}

\author{
Physiotherapeutic intervention in the \\ rehabilitation post sex reassignment surgery \\ male to female: a case report
}

\section{Barbara Rose Bezerra Alves Ferreira' ${ }^{1}$ (1) Flavio Junio do Espirito Santo Carmo da Silva² (1)}

${ }^{1}$ Faculdade Estácio de Alagoas (Maceió). Alagoas, Brasil. babicca@hotmail.com ${ }^{2}$ Autor para correspondência. Faculdade Estácio de Alagoas (Maceió). Alagoas, Brasil. flaviiojuniio@gmail.com

\begin{abstract}
RESUMO | DESCRIÇÃO DO CASO: Este relato de caso de uma mulher transexual, 48 anos de idade, com 15 anos de pós cirurgia de redesignação sexual (CRS) sem queixas. Durante a avaliação inicial foram coletados os dados sociodemográficos, avaliação da dor através da escala visual analógica visual (EVA), a qualidade de vida pelo SF-36, a função sexual pelo QS-F. Foi realizada a avaliação do assoalho pélvico (AP) inspeção, palpação e força seguindo o esquema PERFECT. Após a avaliação foram identificadas estenose vaginal, falta de consciência perineal, fraqueza dos músculos do assoalho pélvico e relatou dor durante a relação sexual. INTERVENÇÃO: Foi sugerido um programa de exercícios através dos dilatadores vaginais, terapia comportamental, treino dos músculos do AP. O programa tinha por objetivo 10 sessões com 40 minutos de duração cada, duas vezes na semana. Ao final do programa foram coletadas a EVA, SF-36 e a avaliação do AP seguindo o esquema PERFECT. Entretanto, o QS-F não foi aplicado ao final do programa proposto devido a inatividade sexual da paciente por 3 meses, inclusive durante o tempo do programa. RESULTADOS: Após a avalição final a paciente apresentou melhora da consciência perineal, penetração via vaginal sem desconforto, entretanto, não houve melhora da força dos músculos do AP. CONSIDERAÇÕES FINAIS: A CRS promove alteração anatômica, podendo ocasionar disfunções urogenitais e/ou sexuais. Neste estudo, a fisioterapia promoveu melhora da conscientização perineal e da estenose vaginal. Com tudo, são necessários mais estudos sobre a fisioterapia nesta população, garantindo assistência e acompanhamento de forma adequada durante este processo, reduzindo suas possíveis complicações tardias.
\end{abstract}

PALAVRAS-CHAVE: Disforia de gênero. Cirurgia de redesignação sexual. Reabilitação. Fisioterapia. Neovagina.

\begin{abstract}
CASE REPORT: This case report of a transsexual woman, 48 years old, with 15 years after sex reassignment surgery (SRS) without complaints. During the initial assessment, socio-demographic data, pain assessment using the visual analog visual scale (VAS), quality of life by SF-36, sexual function by QS-F were collected. The pelvic floor (PF) assessment was performed for inspection, palpation and strength following the PERFECT scheme. After the evaluation, vaginal stenosis, lack of perineal awareness, weakness of the pelvic floor muscles were identified and pain was reported during intercourse. INTERVENTION: An exercise program through vaginal dilators, behavioral therapy, and PF muscle training was suggested. The program aimed at 10 sessions of 40 minutes each, twice a week. At the end of the program, VAS, SF-36 and PF evaluation were collected following the PERFECT scheme. However, the QS-F was not applied at the end of the proposed program due to the patient's sexual inactivity for 3 months, including during the program period. RESULTS: After the final evaluation, the patient showed improvement in perineal awareness, vaginal penetration without discomfort, however, there was no improvement in the strength of the AP muscles. FINAL REMARKS: The SRS promotes anatomical alteration, which can cause urogenital and / or sexual dysfunctions. In this study, physiotherapy promoted improvement in perineal awareness and vaginal stenosis. However, more studies on physiotherapy are needed in this population, ensuring assistance and adequate followup during this process, reducing possible late complications.
\end{abstract}

KEYWORDS: Gender dysphoria. Sex reassignment surgery. Rehabilitation. Physiotherapy. Neovagine. 


\section{Introdução}

A disforia de gênero vem da certeza do indivíduo, do sentimento irreversível, e descontentamento de pertencer ao gênero designado ao nascimento, com isso despertando um forte desejo de modificar-se, na qual muitos passam por uma série de tratamentos hormonais e cirúrgicos, com o intuito de alterar suas características sexuais primarias e secundárias para tornar o seu corpo o mais próximo possível do gênero desejado ${ }^{1,2,3}$.

A cirurgia de redesignação sexual homem-mulher possui técnicas diferentes, porém a mais utilizada é a orquidectomia, que consiste na amputação do pênis, criação da cavidade da neovagina, revestimento da cavidade, reconstrução do hiato uretral, construção dos pequenos lábios e grandes lábios, além da formação clitóris ${ }^{4,5}$.

Ao final do procedimento cirúrgico será colocado molde de espuma de borracha revestido por preservativo. O edema local costuma ser acentuado na primeira semana. A sonda vesical é mantida até o sétimo dia de pós-operatório e o molde deve ser usado durante pelo menos dois meses, sendo relações sexuais vaginais liberadas a partir deste período ${ }^{6,7}$.

A Cirurgia de redesignação sexual pode apresentar grandes problemas em relação ao assoalho pélvico (AP), devido ao fato de várias estruturas importantes do AP serem submetidas a retalhações para que se encaixem "perfeitamente". Entre as complicações relacionadas a CRS vão desde o ato cirúrgico até o pósoperatório seja de forma imediata ou tardia. Dentre elas destacam-se estenose vaginal, estenose uretral, prolapso vaginal, perfuração da parede vaginal, dispareunia e fraqueza da parede vaginal $\left.\right|^{9,10,11}$.

O uso incorreto ou não uso do molde vaginal, que é colocado após o processo cirúrgico pode causar alterações da elasticidade, lubrificação vaginal, fechamento ou estreitamento significativo do canal vaginal formado, secura, e a dor que pode ir se agravando ou gerar disfunções sexuais (dispareunia, dor pélvica crônica, e anorgasmia) ${ }^{12}$.
O papel da fisioterapia dá-se através de técnicas direcionadas ao tratamento das disfunções do assoalho pélvico, pode-se criar propostas terapêuticas com o objetivo de adequação da musculatura pélvica à sua nova inserção e a nova função; orientações e exercícios para manutenção do canal vaginal, ressensibilização e adequação sensorial, promover o incremento funcional desta nova inserção muscular ou visando minimizar as queixas relacionadas às consequências do pós-operatório ${ }^{12}$.

Diante do baixo número de cirurgias de redesignação sexual masculino para feminino, a falta de acompanhamento no pós operatório, o despreparo profissional, a falta de empatia e principalmente a escassez da produção cientifica sobre a intervenção da fisioterapia no pós-operatório de cirurgia de redesignação sexual, este estudo de caso tem como objetivo verificar a intervenção da fisioterapia pós-cirurgia de redesignação sexual homem/mulher, sendo assim, incentivando e despertando o interesse da pesquisa cientifica pelos profissionais a está população.

\section{Medidas de resultados}

A mensuração da dor se deu através de uma escala unidimensional, Escala visual analógica - EVA a qual é a mais utilizada na prática clínica conforme a sua rapidez e aplicação clínica de fácil compreensão em grande parte dos indivíduos. A EVA consiste numa linha com $10 \mathrm{~cm}$ de comprimento, que tem no início da sua escala 0 (ausência de dor), e no final da sua extremidade 10 (dor máxima). A função é marcada na linha com um ponto em que representa a sua dor. Posteriormente, mede-se em centímetros a distância entre o início da linha e o ponto marcado, obtendo-se uma classificação numérica. Quanto maior o escore, maior a intensidade da dor.

A avaliação da qualidade de vida - SF36, que é composto por um questionário multidimensional formado por 36 itens, com 8 domínios (capacidade funcional, aspectos físicos, dor, estado geral da saúde, vitalidade, aspectos sociais, aspectos emocionais e saúde mental) tendo seu escore final obtido por meio de cálculo do Raw Scale de 0 (pior estado geral de saúde) à 100 (corresponde ao melhor estado de saúde). 
A função sexual Q-SF, é um questionário de fácil compreensão e auto aplicado que abrangem em sua avaliação vários domínios (desejo, excitação, orgasmo e seus respectivos correlatos psicofísicos) relacionados às funções sexuais da mulher. O questionário é composto por 10 questões com respostas de 0 a 5 pontos, sendo classificadas de acordo com a intensidade nas categorias ${ }^{13}$.

Quadro 1. Classificação das respostas das perguntas do Q-SF de acordo com a pontuação

\begin{tabular}{|c|c|}
\hline PONTOS & CATEGORIAS \\
\hline 1 & Nunca \\
\hline 2 & Raramente \\
\hline 3 & Aproximadamente $50 \%$ das Vezes \\
\hline 4 & A maioria das Vezes \\
\hline 5 & Sempre \\
\hline
\end{tabular}

Fonte: ABDO, 2006.

O resultado se obtém através da soma das respostas das 10 respostas, multiplicando seu total por dois, o que resulta num índice que varia de 0 a 100. Cálculo do Escore Final: soma dos escores das questões 1, 2, 3, 4, 5, 6, 8, 9 e $10+\left(5\right.$ - escore da questão 7); multiplicar o resultado dessa soma por $2^{13}$.

Quadro 2. Escore final/Resultado/padrão de desempenho sexual Q-SF

\begin{tabular}{|l|l|}
\hline PONTOS & RESULTADO \\
\hline 82 a 100 & Bom a Excelente \\
\hline 62 a 80 & Regular a Bom \\
\hline 40 a 60 & Desfavorável a Regular \\
\hline 0 a 20 & Nulo a Ruim \\
\hline
\end{tabular}

Fonte: ABDO, 2006.

A avalição do assolho pélvico é realizada com o indivíduo em posição ginecológica através da palpação com o intuito de observar as paredes vaginais (pontos dolorosos, tônus muscular, cicatrizes etc.), e os músculos do assoalho pélvico - MAP. A força foi avaliada através da escala de Oxford modificada (que é uma escala subjetiva que classifica o grau de forca muscular do MAP de acordo com a contração voluntária), através do esquema PERFECT (uma escala que avalia a contração muscular, sua intensidade, tempo de sustentação, número de repetições das contrações sustentadas e quantas contrações rápidas consegue realizar). 
Quadro 3. Esquema PERFECT de avaliação funcional subjetiva do assoalho pélvico adaptado

\begin{tabular}{|c|c|c|}
\hline \multirow[t]{8}{*}{$\mathbf{P}=$ Power (força) } & \multicolumn{2}{|c|}{$\begin{array}{l}\text { É avaliada pela contração voluntária máxima e graduada através da Escala de Oxford } \\
\text { Modificada em uma escala de } 0 \text { a } 5 \text {. }\end{array}$} \\
\hline & GRAU & DEFINIÇÃO \\
\hline & 0 & Ausência de resposta muscular \\
\hline & 1 & Esboço de contração não sustentada \\
\hline & 2 & $\begin{array}{l}\text { Presença de contração de pequena intensidade, mas que não } \\
\text { se sustenta. }\end{array}$ \\
\hline & 3 & $\begin{array}{l}\text { Contração moderada, sentida como um aumento da pressão } \\
\text { intravaginal, que comprime os dedos do examinador com } \\
\text { pequena elevação cranial da parede vaginal. }\end{array}$ \\
\hline & 4 & $\begin{array}{l}\text { Contração satisfatória, a que aperta os dedos do examinador } \\
\text { com elevação da parede vaginal em direção a sínfise púbica. }\end{array}$ \\
\hline & 5 & $\begin{array}{l}\text { Contração forte comprime firme os dedos do examinador com } \\
\text { movimento positivo em direção a sínfise púbica. }\end{array}$ \\
\hline $\mathbf{E}=$ Endurance (manutenção) & \multicolumn{2}{|c|}{$\begin{array}{l}\text { Manutenção da contração: corresponde ao tempo, em segundos, com a contração } \\
\text { voluntaria mantida e sustentada, resultado das fibras musculares lentas. Registra-se o } \\
\text { tempo alcançado (no máximo 10s) }\end{array}$} \\
\hline $\mathbf{R}=$ Repetitions (repetições) & \multicolumn{2}{|c|}{$\begin{array}{l}\text { Repetição das contrações mantidas: corresponde ao número de contrações com } \\
\text { sustentação satisfatória, após um período de repouso entre elas. O número sem } \\
\text { comprometimento da intensidade é registrado (no máximo dez repetições) }\end{array}$} \\
\hline $\mathbf{F}=$ Fast (rapidez) & \multicolumn{2}{|c|}{$\begin{array}{l}\text { Número de contrações rápidas: corresponde a medida de contratilidade das fibras } \\
\text { musculares rápidas determinadas após } 2 \text { min de repouso. Anota-se o número de } \\
\text { contrações rápidas de } 1 \mathrm{~s} \text {, sem comprometimento da intensidade. (no máximo } 10 \text { s) }\end{array}$} \\
\hline $\mathbf{E}=$ Every (muitas) & \multirow{3}{*}{\multicolumn{2}{|c|}{ Monitoramento do progresso por meio da cronometragem das contrações. }} \\
\hline $\begin{array}{l}\text { C = Contractions } \\
\text { (contrações) }\end{array}$ & & \\
\hline $\mathbf{T}=$ Timed (cronometragem) & & \\
\hline
\end{tabular}

Fonte; Bo e Larsen, 1990.

\section{Dispositivos de tratamento}

Foram utilizados como instrumentos para o tratamento: Dilatador Vaginal da marca Absoloo: diâmetro 2,5cm; comprimento: 10,9cm; peso: 0,57g; material; Abs e Silicone. (figura 1) Balonete artesanal: uma seringa que em seu bico possui uma sonda enteral № 14 acoplada a um balão (figura 1), e a eletroterapia transvaginal com o aparelho Neurodyn Evolution, modelo N54 da IBRAMED (figura 1), que possui um sonda intracavitário da Ibramed: modelo: Sonda Vaginal; material: Teflon; forma: cilíndrica; dimensões: $21,0 \mathrm{~cm} \times 2,5 \mathrm{~cm} \times 2,5 \mathrm{~cm}(C \times L \times A)$ de uso individual, tendo duas placas de metal (figura 1), na qual era colocada uma camada de gel hidrossolúvel para condução da corrente elétrica e, em seguida, o eletrodo era introduzido no canal vaginal pelo pesquisador.

Foi feita a condução da corrente pulsada bifásica simétrica equilibrada retangular; não polar, a nível motor. frequência $(F)=30 \mathrm{~Hz}$, Largura de pulso $(T)=450 u s$, Tempo de subida= 1, Tempo de descida= 1, ON=4s, OFF= 4s, Tempo de estimulação = 20minutos, para fibras tônicas. 
A amplitude da corrente era aumentada de acordo com a sensação da paciente, na qual era relatada verbalmente pela mesma, começando em $13 \mathrm{~mA}$ e terminando em $40 \mathrm{~mA}$. Ao término de cada sessão o eletrodo intracavitário era higienizado com sabonete antibacteriano e posteriormente guardado em saco ziplock.

O uso da eletroterapia se deu da primeira à última sessão, associada a contração simultânea da paciente. O uso do balonete deu-se na $2^{\circ}$ e $3^{\circ}$ sessão para ensinar e treinar a contração do MAP. Já o dilatador começou a ser utilizado a partir da $4^{\circ}$ sessão.

Figura 1. Dispositivos de Tratamento. (A: Dilatador Vaginal Absoolo; B: Balonete artesanal; C: Neyrodyn Evolution; D: Sonda Vaginal)

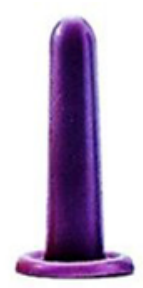

A

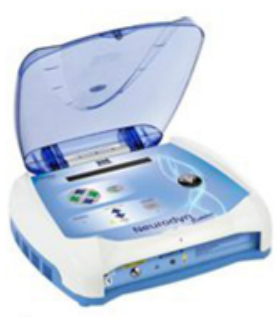

C

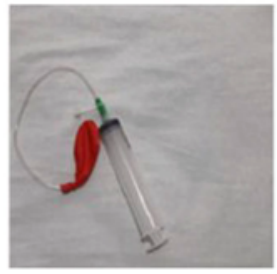

B

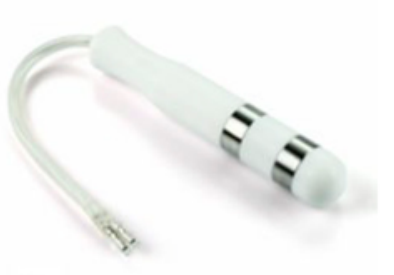

D

\section{Descrição do caso}

O presente estudo ocorreu de acordo com as normas éticas do Conselho Nacional de Saúde quanto à pesquisa envolvendo seres humanos previstas na Resolução 466/2012, de 12 de dezembro de 2012. Foi aprovado pelo Comitê de Ética e Pesquisa da Faculdade Estácio - FAL, sob parecer de número 2.752.542, CAAE 91940018.7.0000.5012 no dia 03/07/2018.

A amostra foi composta por uma transexual mulher, 48 anos de idade, bissexual, parda, 1.69 de altura e $64 \mathrm{~kg}$, ensino médio completo, divorciada, natural do Rio de Janeiro/RJ e procedente de Rio Largo/AL, Militar na reserva, sem queixas, apenas relatava que a ginecologista falou que a vagina estava atrofiada. Em 2003 realizou a CRS no estado de São Paulo, cuja técnica cirúrgica foi a orquedectomia. Durante o pós-operatório fez uso de vibrador por um período de três meses com o objetivo de não fechar o canal vaginal e nem diminuir o comprimento, após esse período relatou que fez extremo esforço físico ao levantar um vaso de plantas sentindo a laceração no local da cirurgia, entretanto não procurou o médico e nem realizou nem um procedimento secundário, também ratifica que está com mais de 1 ano que não vai a ginecologista e que estava a mais de meses sem ter relações sexuais ou penetração via vaginal.

Após ser informada do estudo, e a contribuição da fisioterapia na reabilitação nas disfunções do assoalho pélvico, aceitou participar da pesquisa e assinou o Termo de Consentimento Livre e Esclarecido, sendo encaminhada para a Clínica Escola da Faculdade Estácio de Alagoas, na cidade de Maceió/AL, a qual ofereceu recurso adequado para o desenvolvimento da pesquisa no período de setembro a novembro de 2018. 
As variáveis avaliadas neste relato de caso foram: dor, função sexual e força muscular do assoalho pélvico (AP) e qualidade de vida. Incialmente apresentou: EVA 2, QS-F 58. No exame físico inicial do assoalho pélvico, a paciente apresentou: distância anovulvar de $3 \mathrm{~cm}$, reflexo anocutanio presente, ausência de cicatrizes, ressecamento vaginal, estenose vaginal, falta de consciência perineal. contração dos músculos do assoalho pélvico com uso da musculatura acessória (abdominais, adutores e glúteos) e apneia. Com relação a avaliação dos músculos do assoalho pélvico apresentou um PERFECT inicial de 2/4/5/10. Não apresentou nenhuma disfunção sexual (anorgasmia, vaginismo, dispareunia). Após a avaliação e a explicação sobre a estenose vaginal encontrada, a paciente relatou sentir dor nas últimas relações sexuais e que não conseguia ter uma penetração mais profunda.

As avaliações foram realizadas por um pesquisador que trabalha no campo da fisioterapia saúde da mulher.

De acordo com está avaliação e o diagnóstico de estenose vaginal e fraqueza muscular do assoalho pélvico. O objetivo/expectativa da paciente era ter uma vagina funcional, sendo assim foi proposto um protocolo de dez sessões de intervenção, sendo realizada duas sessões por semana com eletroestimulação e cinesioterapia, e dilatação através dos dilatadores vaginais. Entretanto, a paciente compareceu a 8 sessões das 10 propostas, devido ao seu tempo, as sessões foram realizadas uma vez por semana, com duração de 40 min cada, com foco na estenose vaginal devido o objetivo da paciente.

Na primeira sessão foi realizada a entrevista coletando os dados da paciente, anamnese, avaliação da dor através da escala visual analógica (EVA), qualidade de vida pelo SF-36 e função sexual pelo QSF. Posteriormente foi realizado o exame físico com a paciente em posição ginecológica, com o intuito de observar as paredes vaginais, pontos dolorosos, tônus muscular, atrofia, cicatrizes, e a força dos MAP (mensurada através da escala de Oxford modificada, seguindo o esquema PERFECT), foram introduzidos o dedo médio e indicador no introito vaginal realizando a palpação. Para mensurar força de contração dos músculos do assoalho pélvico os dedos do examinador foram pronados e abduzidos solicitando a participante uma contração voluntária dos músculos do assoalho pélvico, sendo também avaliado o uso da musculatura acessória (glúteo, abdominais, e adutores da coxa) durante a contração. A participante também foi orientada sobre a importância da respiração, da motivação e da contração do MAP para um bom resultado, assim como da anatomia da vulva, e os músculos que formam o assoalho pélvico através de figura ilustrativa.

Na segunda sessão iniciou o treino da contração dos MAP com a paciente em decúbito dorsal, foram realizados: exercícios respiratórios; balonete com $40 \mathrm{ml}$ de água gelada pedindo para a mesma contrair ao comando do examinador e eletroestimulação associada a contração da paciente. Devido ao comprimento do canal vaginal da paciente ser reduzida, a sonda vaginal era expulsa, necessitando que o terapeuta a segurasse até o final da eletroestimulação (figura 3).

Terceira sessão continuou o treino da contração dos MAP.

Na quarta sessão foi iniciado o alongamento vaginal através do dilatador, no tempo de 15 minutos. Os exercícios foram iniciados com molde medindo $21,0 \mathrm{~cm}$ de comprimento e $2,5 \mathrm{~cm}$ de diâmetro, sendo que só era introduzido $5,0 \mathrm{~cm}$, no qual era o suportado pela paciente. Incialmente era alongada as laterais da parede vaginal por um tempo de 1 (um) minuto cada lateral, posteriormente o dilatador era inserido exercendo uma pressão no sentido cranial no introito vaginal até o ponto de desconforto sem dor, feito isso era retraído $2 \mathrm{~cm}$, e depois inserido completamente até o suportado pela paciente. Esse movimento era feito durante 1 minuto, com um descanso de 1 (um) minuto até se iniciar tudo novamente. As dimensões dos moldes progrediram sucessivamente, de acordo com a evolução do túnel vaginal. A paciente foi orientada a fazer o alongamento em casa uma vez por dia por 15 minutos.

Na quinta e na sexta sessão repetiu-se o alongamento vaginal, e após colocou-se o dilatador no canal vaginal exercendo uma pressão no sentido cranial no introito vaginal até o ponto de desconforto sem dor. A pressão era mantida por 20 minutos sem fazer movimentos.

A partir da sexta sessão a paciente foi orientada a dormir com o dilatador diariamente, com o auxílio de um esparadrapo de microporen (antialérgico) para conter o dilatador dentro do canal vaginal. Iniciando com o de $10,09 \mathrm{~cm}$ de comprimento e $2,5 \mathrm{~cm}$ de diâmetro. 
$\mathrm{Na} 7^{\circ}$, e $8^{\circ}$ sessões a conduta se manteve a mesma da 5 sessão e a orientação de fazer uso do dilatador durante o período de sono noturno.

O programa teve uma duração total de oito semanas e o paciente não relatou desconforto ou dificuldade em utilizar o dispositivo. Ao Final do programa foram avaliadas novamente todas as variáveis, descritos na tabela 2.

\section{Resultado}

O programa de tratamento proposto para tratar a estenose vaginal de forma conservadora através dos dilatadores vaginais no total de 8 semanas/8 sessões obteve resultado satisfatórios, podendo ser observado na tabela 1.

Tabela 1. Dados relacionados ao comprimento vaginal antes e depois o tratamento

\begin{tabular}{lcc}
\hline Tabela 1: SESSÕES & ANTES & APÒS \\
\hline $4^{\circ}$ Sessão & $5 \mathrm{~cm}$ & $6,0 \mathrm{~cm}$ \\
$5^{\circ}$ Sessão & $5 \mathrm{~cm}$ & $7,7 \mathrm{~cm}$ \\
\hline $6^{\circ}$ Sessão & $5,5 \mathrm{~cm}$ & $10,9 \mathrm{~cm}$ \\
$7^{\circ}$ Sessão & $5 \mathrm{~cm}$ & $10,9 \mathrm{~cm}$ \\
$8^{\circ}$ Sessão & $5,8 \mathrm{~cm}$ & $10,9 \mathrm{~cm}$ \\
\hline
\end{tabular}

Na tabela 1 podemos observar todas as medidas do comprimento do canal vaginal da paciente. As medidas eram realizadas antes e no final da sessão por meio do dilatador vaginal. O dilatador era inserido até encontrar uma resistência, feito isso o mesmo era retirado e medido por meio de fita métrica.

Podemos observar que antes das sessões o comprimento do canal vaginal é de $5 \mathrm{~cm}$, e que somente na $6^{\circ}$ e na $8^{\circ}$ sessão podemos constatar uma mudança nos valores antes da conduta fisioterapêutica. Este aumento ocorreu devido aos exercícios domiciliares realizados pela paciente. Portanto, na semana em que a paciente fazia os exercícios houve uma distensibilidade no comprimento do canal vaginal. Então somente com as oito sessões foram obtidos como resposta inicial e final respectivamente os valores de 5,0 cm e 10,9cm, um aumento de 5,9 $\mathrm{cm}$. Entretanto esses valores não apresentam uma evolução satisfatória já que esses valores são verificados no decorrer da sessão através dos dilatadores vaginais.

Figura 2. Mensuração da profundidade do canal vaginal. (A: Profundidade Inicial 5cm; B: No decorrer do tratamento 7,7cm; C: Profundidade Final 10,9cm)

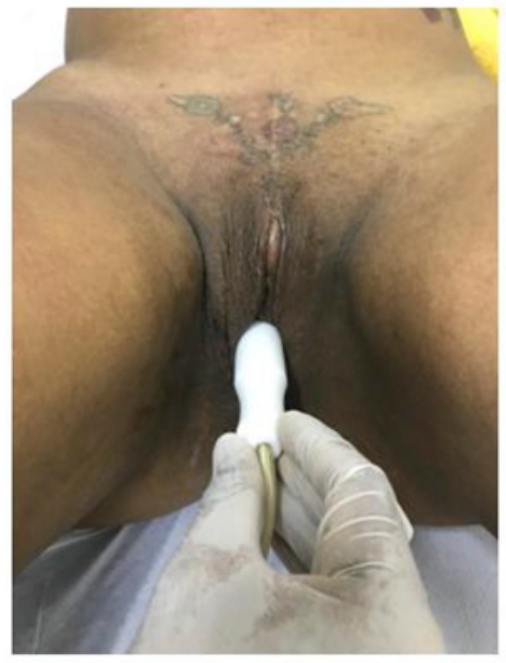

A

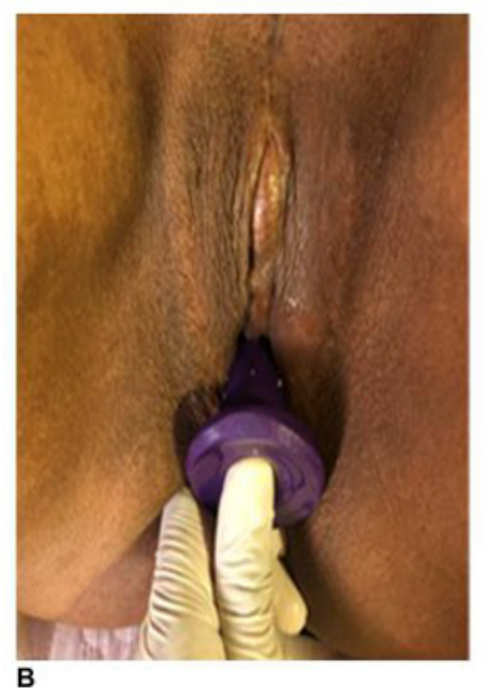

B

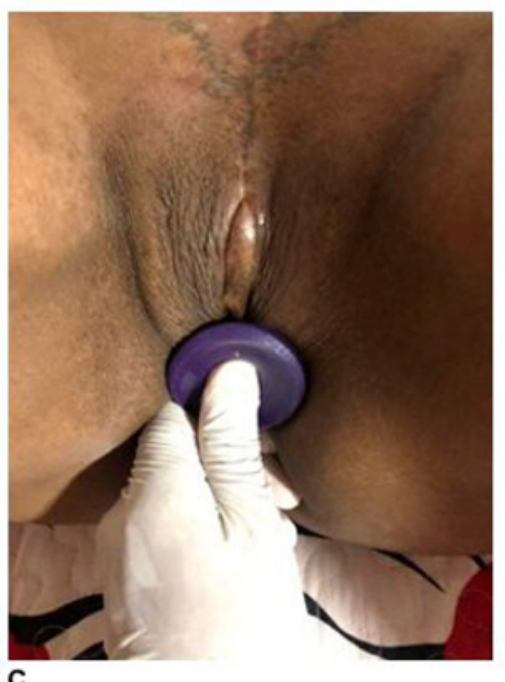

C 


\begin{tabular}{lcc}
\hline \multicolumn{1}{c}{ COLETA DE DADOS DA PESQUISA } & INICIAL & FINAL \\
& & \\
\hline EVA & 2 & 0 \\
\hline QS-F & 58 & Ausência sexual \\
PERFECT & $2 / 4 / 5 / 1 / 0$ & $2 / 4 / 5 / 1 / 0$ \\
& & \\
\hline
\end{tabular}

Foi identificado durante os exercícios que a dor estava relacionada quando a paciente tentava uma penetração sexual mais profunda. Durante os exercícios de dilatação vaginal, a paciente relatava desconforto/dor, que era tolerado por ela, e ia aliviando após os exercícios. A dor foi quantificada de acordo com a EVA o resultado de 2. No final do programa a paciente apresentou uma diminuição total da dor (tabela 2), devido ao aumento do comprimento vaginal (tabela 1).

Com relação a avaliação da função sexual da paciente um aspecto fundamental na presente pesquisa, que é avaliado através do QS-F, foi obtido de forma inicial o escore de 58 pontos (tabela 2). De acordo com o próprio Questionário de função sexual significa bom a excelente. Com tudo, na avaliação final não foi aplicado o questionário devido a inatividade sexual da paciente por mais de 6 meses, inclusive durante o programa de tratamento (tabela 2).

Quanto à avaliação da qualidade de vida (tabela 3), os componentes do questionário SF-36 são avaliados através de escores que variam de 0 a 100, no qual 0 é o pior estado e 100 o melhor. Após o tratamento observamos índices que indicam melhora, sobretudo em relação aos aspectos social e estado geral de saúde que atingiram $100 \%$, os domínios "aspectos emocionais" e "aspectos físicos' se manteve com pontuação máxima tanto no início quanto no término do tratamento do SF-36. Outro aspecto que podemos observar melhora foi a dor, houve uma diminuição com relação ao pré-tratamento, cujo escore inicial pra o item alcançou $61 \%$, tendo uma redução de $11 \%$ em comparação com o pré-tratamento. Entretanto houve uma diminuição nos scores da vitalidade (- 30\%), e saúde mental (-8\%) da paciente.

No presente estudo não foi verificado nenhum efeito colateral em relação aos recursos utilizados.

Tabela 3. Dados relacionados a qualidade de vida antes e depois o tratamento

\begin{tabular}{lcc}
\hline Itens & Pré-Tratamento (\%) & Pós-Tratamento (\%) \\
Capacidade funcional & 85 & 90 \\
Aspectos físicos & 100 & 100 \\
Dor & 72 & 61 \\
Estado geral de saúde & 52 & 100 \\
Vitalidade & 85 & 55 \\
Aspectos sociais & 88 & 100 \\
Aspectos emocionais & 100 & 100 \\
Saúde mental & 68 & 60 \\
\hline
\end{tabular}




\section{Discussão}

Os níveis de evidencia referentes a CRS são muitos baixos, entretanto as complicações cirúrgicas neste tipo de intervenção são numerosas e muito frequente, sendo bem descritas no estudos realizado por Franco et al. ${ }^{7}$ Krege $^{5}$. Chaudhary ${ }^{14}$. Na qual afirmam que entre as complicações tardias relacionadas a cirurgia de redesignação sexual destacam-se as relacionas à região genital, ao trato urinário, a eventos gastrointestinais, a distúrbios da cicatrização e eventos inespecíficos, sendo a estenose vaginal a mais encontrada. Assim também indo de acordo com o estudo realizado por Neto et al. ${ }^{10}$ na qual constatou que a perda do comprimento vaginal foi encontrada em 25 casos que correspondem a $8 \%$ e que as mulheres foram submetidos a uma nova intervenção cirúrgica.

Silva et al. ${ }^{19}$ consideram a estenose vaginal como o estreitamento e/ou encurtamento do canal vaginal interferindo no uso de absorventes internos, função sexual, exame ginecológico/físico. Deans et al. ${ }^{20}$ asseveram que uma neovagina deve ter um diâmetro e um comprimento adequado para permitir penetração, Neto et al. ${ }^{10}$ Chaudhary et al..$^{14}$ asseguram que esse comprimento gira em torno de $12-14 \mathrm{~cm}$.

No presente estudo foi constatado a dificuldade da paciente em contrair o assoalho pélvico com o comando verbal do fisioterapeuta, mesmo sendo encorajada ("vamos você consegue", 'isso aí", "contrai, mantém a contração, relaxa", "respira", "puxa os anus para cima", "contrai como se fosse prender um pum", com isto, sendo necessário o uso do balonete e da eletroterapia para ajudar a paciente aprender a contrair o MAP.

Denton e Maher ${ }^{17}$ afirmam que é relativamente comum encontrar mulheres que não saibam contrair os músculos do assoalho pélvicos, tanto jovens como adultos, são incapazes de fazê-lo espontaneamente pela falta de consciência sinestésica dessa região. Moreno $^{18}$. afirma que dentro dos recursos da fisioterapia está a eletroterapia que é um importante componente para fortalecimento da MAP e também como estímulo para despertar a consciência corporal da maneira correta de se contrair, função está a qual é muitas vezes utilizada durante a avaliação, especialmente em mulheres que não conseguem contrair esses músculos pelo comando verbal e orientação do fisioterapeuta.
De acordo com um estudo de Piassarolli et al. ${ }^{15}$ Avaliando o efeito do treinamento muscular do assoalho pélvico ( cinesioterapia), em 26 mulheres diagnosticadas com disfunção sexual (excitação, orgasmo e dispareunia), que foram submetidos a dez sessões (uma ou duas vezes por semana, com duração de 50 minutos), sendo avaliados antes do tratamento, após cinco sessões e no final do tratamento por via vaginal bidigital, palpação (avaliação da força muscular através do esquema PERFECT), eletromiografia intravaginal e avaliação da qualidade de vida sexual por meio do índice de função sexual feminina. $O$ estudo concluiu que o treinamento muscular do assoalho pélvico, melhora a força muscular (69\% das mulheres apresentaram nota 4 ou 5 na avaliação final), e melhora total das queixas sexuais. Portanto foi possível obter através do treino do MAP uma melhora na função sexual, na força da MAP, da dispareunia, aumento da lubrificação e intensidade do orgasmo.

Embora não seja possível obter na literatura estudos que mostrem ou relatem o papel da Fisioterapia de forma imediata ou tardia na reabilitação pós cirurgia de redesignação sexual homemimulher, tão pouco estudos que descreva ou avalie os aspectos físicos, sociais e emocionais após o processo cirúrgico. Franco et al. ${ }^{7}$ Freitas et al. ${ }^{12}$ Corroboram que são várias as complicações associadas a esses procedimentos que são semelhantes as tratadas atualmente pela fisioterapia de forma satisfatória.

A terapêutica proposta para tratar a estenose vaginal se deu de forma conservadora através do uso de dilatadores vaginais, cujo o objetivo é tratar a estenose vaginal criando uma vagina anatômica e funcional que proporcione uma vida sexual ativa e um bem-estar psicológico satisfatório, através do alongamento e dilatação progressiva do canal. Método esse usado em outros estudos por Piazza21. Chaudhary et al. ${ }^{14} \mathrm{e}$ Gupta ${ }^{22}$. Na qual Ratificam que o método Frank ( é um método usado para tratar a estenose vaginal de forma conservadora, que consiste na realização de exercícios diários através de dilatadores associados a manobras compressivas e dilatadoras sobre o introito vaginal por no mínimo 15 minutos, entre uma a três vezes ao dia, assim produzindo pressão progressiva e aumentando a flexibilidade da parede vaginal permitindo que esta aumente de comprimento e largura) é eficaz para tratar a estenose vaginal, ganhando comprimento e diâmetro através dos dilatadores, esses são aumentados progressivamente, sendo possível em algumas sema- 
nas obter uma vagina de profundidade suficiente para que a paciente possa ativar sua vida sexual.

Carvalho et al. ${ }^{2}$ avaliaram em seu estudo que 7 pacientes (25\%), evoluíram com estenose precoce da neovagina atribuída ao uso inadequado do molde vaginal no período pós-operatório inicial, 6 delas realizou uma nova neovaginoplastia e uma paciente optou por não se submeter à intervenção, e nos primeiros 4 meses uma paciente perdeu continuidade no pós-operatório, evoluindo com encurtamento da neovagina $(3,0 \mathrm{~cm})$ e dispareunia, sendo orientada a realizar exercícios de pressão com os dilatadores pelo método Frank. Também indo de acordo Marin et al. ${ }^{23}$ analisaram dez mulheres submetidas ao tratamento com dilatadores pela técnica de Frank, e ratifica a eficácia da técnica de dilatação na estenose vaginal, obtendo um resultado de comprimento inicial $2,4 \pm 2,0 \mathrm{~cm}$, e final de $6,9 \pm 1,1 \mathrm{~cm}$, um $p<0,0001$ ).

Corroborando ainda mais com os benefícios da fisioterapia e a eficácia da técnica de dilatação por meio dos dilatadores vaginais na estenose vaginal, uma revisão sistemática realizada por Denton e Maher. ${ }^{16}$ sobre as intervenções realizadas por fisioterapeutas, na estenose vaginal, pós-radioterapia pélvica no tratamento do câncer do colo do útero, foram encontrados os benefícios de duas técnicas aplicadas pelos fisioterapeutas (dilatadores e terapia manual, especificamente pressão digital), os autores afirmam que a técnica de dilatação duas vezes por dia durante quatro a oito semanas promove a redução da estenose, facilitando o retorno das mulheres as suas atividades sexuais, meIhorando assim sua autoestima e autoconfiança.

No presente estudo a voluntária foi informada sobre a importância de realizar os exercícios domiciliares de alongamentos na neovagina e o uso dos dilatadores durante o período de sono noturno para um bom progresso e resultado, do mesmo modo como a importância de está motivada e entusiasma para um bom progresso da técnica.

Entretanto, apesar de sempre está sendo lembrada, orientada e acompanhada pessoalmente e por WhatsApp sobre a importância dos exercícios de dilatação para manter a vagina funcional, de realizar os exercícios domiciliares, se tinha dúvidas para realização dos exercícios domiciliares, se estava realizando os exercícios domiciliares ou se realizou no dia, a mesma devido suas atividades diárias (trabalho, filhos e tarefas de casa) informou que não realizava os exercícios diariamente pois encontrava-se sem tempo, cansada para realizar as atividades além dos atendimentos fisioterapêuticos e que as vezes esquecia de realizar os exercícios domiciliares, com isto, só realizou os exercícios de alongamentos e a dilatação apenas 2 dias com duração de 20 minutos devido esquecimento e falta de tempo. Já o uso do dilatador para dormir foi usado apenas nos dias que era feito o atendimento (começando da sexta sessão) devido relatar não ter ninguém para colocar o dilatador para ela dormir durante a semana, sendo que ela foi treinada a colocar o dilatador.

A voluntária também foi encaminhada a psicóloga para um melhor acompanhamento, e entendimento para a adesão ao tratamento (se ela estava fazendo por vontade própria ou pelo parceiro) devido ao resultados da técnica também está associado ao empenho e dedicaçao da paciente. A voluntaria relatou que não era necessário e que não teria disponibilidade para ir as consultas.

Fisher et al. ${ }^{24}$ Corrobora que esta não adesão pode estar relacionada a três principais fatores: informação, motivação e comportamento. Em um estudo realizado por Bakker et al. ${ }^{25}$ avaliando relatos quantitativos com relação aos determinantes das pacientes ao uso de dilatadores vaginais após radioterapia pélvica, dois recentes estudos buscam sanar as barreiras ao uso do dilatador. Em um estudo com 30 mulheres, na qual foram recomendadas fazerem uso de dilatadores vaginais, as pacientes relataram falta de tempo ou privacidade, esquecimento ou cansaço como motivos para a não adesão. Outros fatores determinantes para o uso dos dilatadores foram: design de plastico rígido do dilatador na qual despertou emoções negativas e medo antecipado da dor.

Gupta $^{22}$ afirma que, o tratamento da estenose da neovagina pode ser administrada pela paciente quando essa for orientada corretamente, entretanto o resultado positivo da técnica irá depender da motivação da paciente em realizar as atividades propostas, e que cerca de $90-96 \%$ dos pacientes conseguiram o sucesso anatômico e funcional por dilatação vaginal. O comprimento vaginal só será mantido/aumentado se o indivíduo continuar a dilatação em casa ou tiver relação sexual no mínimo duas vezes na semana com penetração na neovagina, caso pare a dilatação não se machucará em uma relação sexual, mas pode precisar retomar a dilatação antes da atividade sexual no futuro. 
Há uma escassez de estudos na literatura que avalie a qualidade de vida de pessoas que passaram pela cirurgia de redesignação sexual tanto pré como pós-tratamento, entretanto, sabemos que este processo é algo esperado por muitos transexuais, na qual muitos sentem repudio pelo seu órgão genital biológico, desta forma já interferindo na sua saúde física, mental e nas relações interpessoais.

A forte expectativa e ansiedade com relação a CRS acaba interferindo na qualidade de vida do paciente, seja de forma positiva ou negativa, contudo, a cirurgia pode apresentar complicações (durante o ato cirúrgico ou no pós-operatório), na qual é necessário outras intervenções cirúrgicas ou tratamento fisioterapêutico, desta forma rompendo com as expectativas do paciente e repercutindo de forma negativa na sua qualidade de vida. Neto et al. ${ }^{10}$ afirmam que as repercussões pós-cirúrgicas impactam negativamente na saúde mental e sexual dos transexuais.

Portanto se faz necessário que os profissionais da saúde e pesquisadores, prestem um atendimento humanizado, com ausculta qualificada para as expectativas destes pacientes, sendo assim, dando-lhes explicações sobre a cirurgia, possíveis complicações e se dispondo a prestar um acompanhamento ainda melhor no pós-operatório, avaliando a qualidade de vida destes paciente tanto antes como após a cirurgia.

Diante dos nossos resultados podemos observar que houve uma melhora de $48 \%$ no estado geral de saúde da paciente, com isto, podemos concluir que a função sexual está muito além do funcional sendo necessário sempre avaliar todos os aspectos físicos, sociais e mentais.

Outro aspecto observado foi a dor referida pela paciente durante a relação sexual, isto acontecia quando o parceiro tentava uma penetração mais profunda, o mesmo aconteceu durante os exercícios de dilatação, conforme ia progredindo a paciente relatava desconforto, mas de acordo com a mesma era uma dor suportável (EVA 2) e que não chegava nem perto da dor que o parceiro a causava.

Ao final do tratamento a paciente relatou está supersatisfeita, empolgada e motivada com a possibilidade de ter uma vagina funcional e ativa novamente e que procuraria sempre separar um tempo para ela ir a ginecologista e realizar os exercícios, já que ela ganhou o kit com dilatadores. A paciente foi informada mais uma vez sobre a necessidade e a importância da realização dos exercícios diariamente e orientada a como realizá-los e que estaríamos disponíveis para qualquer dúvida.

Ressalto ainda a importância da realização de mais pesquisas devido a escassez de estudos que avalie a associação entre uso do dilatador, comprimento vaginal e função sexual, dificultam um discursão ainda mais robustas.

O estudo apresenta limitações como o fato de ser um relato de caso, no qual não é possível excluir algumas variáveis de confundimento existentes. Outra limitação é que poderia ser usado um exame de imagem, por exemplo: ressonância magnética, para avaliar com precisão a profundidadelcomprimento anatômico do canal vaginal da paciente. A falta de tempo da paciente para realizar os exercícios domiciliários foi outra limitação encontrada, pois poderia ser observado com mais precisão se os ganhos seriam mantidos.

Este estudo de caso corrobora que a fisioterapia pode e deve atuar no pós operatório de CRS. Uma vez que o fisioterapeuta através de suas técnicas e recursos fazem uso dos vaginais, eletroestimulação, terapia comportamental em outras patologias semelhantes a complicações da CRS. O presente estudo traz bons resultados no tratamento da estones vaginal com uso da dilatação progressiva, obtendo uma distensibilidade de $5,9 \mathrm{~cm}$ no comprimento vaginal.

\section{Conclusão}

Este estudo de caso, apresenta melhora clínica e compensação das possíveis complicações tardia pós-operatório CRS. Apesar dos resultados positivos do estudo, mostrando que fisioterapia através de seus recursos pode atuar de forma positiva nestas complicações, são necessários mais estudos sobre a fisioterapia nesta população, garantindo assistência e acompanhamento de forma adequada durante este processo, reduzindo suas possíveis complicações tardias. 


\section{Contribuições dos autores}

Ambos os autores contribuíram igualmente de todas as etapas deste relato.

\section{Conflitos de interesses}

Nenhum conflito financeiro, legal ou político envolvendo terceiros (governo, empresas e fundações privadas etc.) foi declarado para nenhum aspecto do trabalho submetido (incluindo, mas não se limitando a subvenções e financiamentos, participação em conselho consultivo, desenho de estudo, preparação de manuscrito, análise estatística, etc.).

\section{Referências}

1. Arán M. A transexualidade e a gramática normativa do sistema sexo-gênero. Ágora Estud em Teor Psicanalítica. 2006;9(1):49-63. doi: 10.1590/S1516-14982006000100004

2. Carvalho BR, Reis RM, Moura MD, Lara LAS, Nogueira AA, Ferriani RA. Neovaginoplastia com membrana amniótica na síndrome de Mayer-Rokitansky-Küster-Hauser. Rev Bras Ginecol Obs. 2007;29(12):619-624. doi: 10.1590/S010072032007001200004

3. Peres WS, Toledo LG. Dissidências existenciais de gênero: resistências e enfrentamentos ao biopoder. Rev Psicol Política. 2011;11(22):361-277.

4. Selvaggi G, Dhejne C, Landen M, Elander A. The 2011 WPATH Standards of Care and Penile Reconstruction in Female-toMale Transsexual Individuals. Adv Urol. 2012;2012:581712. doi: $10.1155 / 2012 / 581712$

5. Krege S, Bex A, Lümmen G, Rübben H. Male-to-female transsexualism: A technique, results and long-term follow-up in 66 patients. BJU Int. 2001;88(4):396-402. doi: 10.1046/j.1464410X.2001.02323.x

6. Jarolím L, Šedý J, Schmidt M, Naňka O, Foltán R, Kawaciuk I. Gender reassignment surgery in male-to-female transsexualism: A retrospective 3-month follow-up study with anatomical remarks. J Sex Med. 2009;6(6):1635-1644. doi: 10.1111/j.1743$\underline{6109.2009 .01245 . x}$

7. Franco T, Miranda LC, Franco D, Zaidhaft S, Aran M. Transgenitalização masculino / feminino: experiência do Hospital Universitário da UFRJ. Rev Col Bras Cir. 2010;37(6):426-434. doi: 10.1590/S0100-69912010000600009

8. Amend B, Seibold J, Toomey P, Stenzl A, Sievert KD. Surgical reconstruction for male-to-female sex reassignment. Eur Urol. 2013;64(1):1-9. doi: 10.1016/j.eururo.2012.12.030
9. Cooperberg MR, Vickers AJ, Broering JM, Carroll PR. Comparative risk-adjusted mortality outcomes after primary surgery, radiotherapy, or androgen-deprivation therapy for localized prostate cancer. Cancer. 2011;117(12):2825. doi: 10.1002/cncr.25456

10. Rosi Neto R, Hintz F, Krege S, Rübben H, Vom DF. Gender reassignment surgery - A 13 year review of surgical outcomes. Int Braz J Urol. 2012;38(1):97-107. doi: 10.1590/S1677$\underline{55382012000100014}$

11. Martins KLD, Oliveira PS. Função miccional, evacuatória e sexual de mulheres transexuais após cirurgia de redesignação sexual [monografia]. Brasília: Faculdade de Ciências da Educação e Saúde Faces; 2018.

12. Freitas TEF, Andrade LCC, Ferreira LA. O papel da fisioterapia no pós-operatório de neovaginoplastia. Revista da Sogia. 2015;16(3):12-17.

13. Abdo CHN. Elaboração e validação do quociente sexual Versão feminina: Uma escala para avaliar a função sexual da mulher. Rev Bras Med. 2006;63(9):477-482.

14. Chaudhary R, Dhama V, Singh S, Azad R. Vaginoplasty in mayer Rokitansky-Kuster-Hauser syndrome using amnion: a case series. Int J Reprod Contraception, Obstet Gynecol. 2016;5(11):3832-3839. doi: $10.18203 / 2320-1770 . i j r c o g 20163849$

15. Piassarolli VP, Hardy E, Andrade NF, Ferreira NO, Osis MJD. Treinamento dos músculos do assoalho pélvico nas disfunções sexuais femininas. Rev Bras Ginecol Obs. 2010;32(5):234-240. doi: 10.1590/S0100-72032010000500006

16. Denton AS, Maher EJ. Interventions for the physical aspects of sexual dysfunction in women following pelvic radiotherapy. Cochrane Database Syst Rev. 2003;(1). doi: 10.1002/14651858. CD003750

17. Grosse D, Sengler J. Reeducação Perineal. 1.ed. São Paulo: Manole; 2002.

18. Moreno LA. Fisioterapia em Uroginecologia. 2.ed. Rio de Janeiro; 2008.

19. Silva RDN, Rosa LM, Radünz V, Cesconetto D. Avaliação e classificação da estenose vaginal na braquiterapia: Validação de conteúdo de instrumento para enfermeiros. Texto Context - Enferm. 2018;27(2):e5700016. doi: 10.1590/0104$\underline{070720180005700016}$

20. Deans R, Berra M, Creighton SM. Management of Vaginal Hypoplasia in Disorders of Sexual Development: Surgical and Non-Surgical Options. Sex Dev. 2010;4(4-5):292-299. doi: $\underline{10.1159 / 000316231}$

21. Piazza MJ. Neovaginoplastias : uma revisão dos diversos métodos de tratamento. Feminina. 2014;42(3):121-127. 
22. Gupta SM. ACOG Committee Opinion No. 728. Obstet Gynecol. 2018;131(1):35-42. doi: 10.1097/AOG.0000000000002458

23. Marin JS, Lara LAS, Silva AV, Reis RM, Junqueira FR, Rosae-Silva ACJS. Tratamento cirúrgico e conservador da agenesia vaginal : análise de uma série de casos. Rev Bras Ginecol Obs. 2012;34(6):274-277. doi: 10.1590/S0100-72032012000600006

24. Fisher JD, Fisher WD. The information-motivation behavioral skills model. In: DiClemente RJ, Crosby RA, Kegler MC, editors. Emerging Theories in Health Promotion Practice and Research: Strategies for Improving Public Health. Indianapolis; 2002. P. 4070.

25. Bakker RM, Vermeer WM, Creutzberg CL, Mens JW, Nout RA, Ter Kuile MM. Qualitative accounts of patients' determinants of vaginal dilator use after pelvic radiotherapy. J Sex Med.

2015;12(3):764-773. doi: 10.1111/jsm.12776 